\title{
Marco geológico e hidrológico de la mega-fauna fósil del pleistoceno, de la porción meridional del Estado de Hidalgo, México central
}

\author{
L. E. Ortiz Hernández $z^{1,2^{\star}}$, J. C. Escamilla Casas²
}

${ }^{1}$ ESIA-Unidad Ticomán, Instituto Politécnico Nacional. Ticomán No. 600, Col San José Ticomán Ciudad de México, C.P.07340, México. leoh44@hotmail.com

2Área Académica de Ciencias de la Tierra y Materiales Universidad Autónoma del Estado de Hidalgo. Carretera Pachuca-Tulancingo km 4.5, Pachuca, Hidalgo, C.P. 42184, México. pepino31@hotmail.com

*Autor de correspondencia: leoh44@hotmail.com

\section{RESUMEN}

El marco geológico e hidrológico de la mega-fauna fósil del Pleistoceno (Mamuth, Equus $s p$. y Glyptodon) de la porción meridional del estado de Hidalgo (región EpazoyucanTecocomulco), es referido a un análisis de la distribución de los cauces hídricos remanentes y la red de drenaje actual, que muestra patrones de tipo radial centrífugo, dendrítico, subdendrítico, paralelo y sub-paralelo, correspondiente a la litología (rocas volcánicas y piroclásticas de tipo básico, intermedio y ácido) y sedimentos fluviales y lacustres del Pleistoceno tardío, así como al tipo de estructuras volcánicas presentes como son flujos de lava, flujos piroclásticos, domos, conos cineríticos, volcanes escudo y calderas del Mioceno al Pleistoceno-Cuaternario.

El análisis permite establecer que la estructura geológica regional (en bloques fallados) y la cercanía a grandes cuerpos de agua, fueron propicios al desarrollo de la mega-fauna fósil en esta región de México central.

Palabras Clave: Mega-fauna, Pleistoceno, Marco geológico-hidrológico, Hidalgo, México.

\section{INTRODUCCIÓN}

El Estado de Hidalgo, en su porción meridional, constituye el armazón geológico del sector oriental de la Faja Volcánica Transmexicana (FVT) (Figura 1), que consiste en un extenso mosaico de campos volcánicos del Mioceno y del Plioceno-Cuaternario.

La mega-fauna fósil localizada en la porción meridional del estado de Hidalgo, en México central (región Epazoyucan-Tecocomulco) (Figura 1), incluye los organismos de gran talla como mamut, caballo mexicano y armadillo gigante del Pleistoceno tardío (Rancholabreano) [2 y 3]. Dicha fauna fósil se localiza específicamente en:

El ejido Matías Rodríguez, municipio de Singuilucan, dónde se encontró una mandíbula de Mammuthus columbi, del Pleistoceno tardío [4] y otros restos óseos como colmillos, costillas, columna vertebral y pelvis [5], alojados en sedimentos fluviales.

En las barrancas Piedras Negras, El Berrendo y San Agustín, cerca de Epazoyucan, se describe mega-fauna fósil (Equus conversidens o caballo mexicano y Gliptodontes o armadillo gigante) [2 y 3], en una secuencia de abanicos aluviales y rellenos de valle, conformada por aluvión y grava volcánica.

En Ventoquipa, en el municipio de Santiago Tlapacoya, se señala la ocurrencia de restos de mastodonte americano (Mammut americanum), del Pleistoceno tardío, asociado a 
molar de Equus corvensidens y vértebras de Bison sp., que se encontraron en sedimentos de grava, arena y arcilla depositados en un ambiente fluvial [6].

En San Jerónimo, cerca de Tepeapulco, se reporta una secuencia estratigráfica de 600 $m$ de espesor del Pleistoceno, en donde a la base se encontró depósitos lacustres: grava y limo de origen volcaniclástico, con fragmentos de andesita basáltica y restos de Mammuthus columbi y Equus sp. [7].

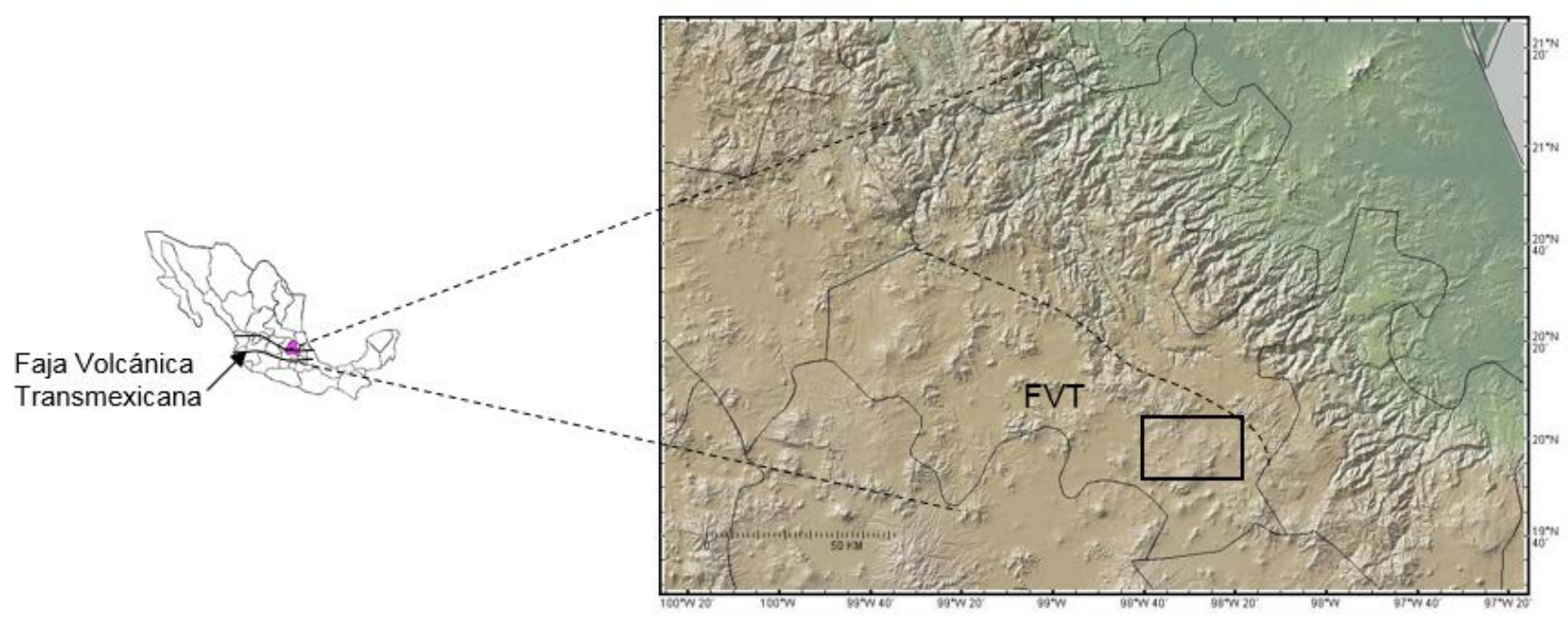

Figura 1. Localización de la porción meridional (cuadro) del estado de Hidalgo, en la Faja Volcánica Transmexicana (FVT). Mapa tomado de [1] (modificado).

\section{MARCO TEÓRICO}

Varios complejos volcánicos que van del Mioceno al Pleistoceno-Cuaternario afloran en el área, entre los que se tiene:

Campo volcánico de Epazoyucan-Singuilucan, constituido por domos dacíticos, brechas volcánicas dacíticas, ignimbritas (22.6ะ0.66 Ma; U/Pb en zircón [8]), vitrófidos y depósitos de flujos de bloques y cenizas de composición riolítica del Mioceno [9 y 10]. Después de un hiatus magmático de $\sim 7 \mathrm{Ma}$ [11 y 8], se tiene un vulcanismo bimodal representado por pómez relacionada al complejo estratovolcánico riolítico peralcalino del Pleistoceno de la sierra de Las Navajas [12 y 13], constituido de derrames de lava y domos con lentes de obsidiana, depósitos piroclásticos de avalancha-brechas y tobas, así como rocas epiclásticas. Este complejo sobreyace discordantemente a lavas andesíticas y dacíticas del Grupo Pachuca [14]. Rocas basálticas emplazadas en conos de escoria y flujos de lava [9], de edad radiométrica K/Ar 1.8 y 2.58ะ0.15 Ma [12 y 15], son parte de este vulcanismo bimodal.

Campo volcánico de Apan-Tezontepec [16 y 17], compuesto de conos de escoria, volcanes escudo y domos alineados al NE-SO de composición basáltico-andesítica, del Pleistoceno. Hay evidencia de actividad volcánica intermedia-ácida del Mioceno mediotardío (13.4-5.3 Ma, K/Ar) [16], siendo la andesita Apan la unidad más antigua. JuárezLópez (2015) [18] denomina andesita Chichicuautla, a una secuencia volcánica posterior datada en $9.82 \pm 0.21 \mathrm{Ma}(\mathrm{K} / \mathrm{Ar})$. 
Campo volcánico Xihuingo-La Paila [19], consiste de conos de escoria con flujos de lava asociados, volcanes escudo, domos dacíticos y depósitos piroclásticos-ignimbritas, emplazados en dos eventos: uno del Mioceno Medio-Tardío ( 13.4-9.8 Ma) con predominio de domos y depósitos piroclásticos andesíticos a riolíticos; otro del Pleistoceno, representado por conos monogenéticos y volcanes escudo de composición máfica y un complejo de domos silícicos, con edades $\mathrm{K} / \mathrm{Ar}$ entre $\sim 2.7$ a $0.18 \mathrm{Ma}$ [19]. El complejo de domos El Yolo, es un campo volcánico silícico (riodacita y riolita) pliocénico a pleistocénico, asociado con flujos piroclásticos, cuyo afloramiento se extiende en la porción oriental de la ciudad de Tulancingo, NE de Cuautepec y O de Acaxochitlán. LópezHernández (2009) [20] interpreta este vulcanismo, como parte de una gran caldera de 32 $\mathrm{km}$ de diámetro (caldera Tulancingo) del Plioceno tardío ( -3.0 a $2.7 \mathrm{Ma}[20]$ ) y de una caldera de $18 \mathrm{~km}$ de diámetro (caldera Acoculco), del Plioceno tardío-Pleistoceno (2.7$0.06 \mathrm{Ma}$ [21]), anidada en la primera [20]. Estas produjeron lavas dacíticas a riodacíticas e ignimbritas, culminando con el emplazamiento de domos riodacíticos, flujos piroclásticos y basaltos.

Los sedimentos dónde se hallan los restos de la mega-fauna fósil son fluviales o lacustres del Pleistoceno tardío, y correlacionables con la Formación Tarango, si bien HuizarÁlvarez et al. (2001) [22], la denominan como Formación Calpulalpan [23], en la semifosa de Tecocomulco.

\section{PARTE EXPERIMENTAL Y RESULTADOS}

La metodología de trabajo consistió en el análisis de la información geológica actualizada sobre el área de estudio, dado que se trata de campos volcánicos extensos, cada uno con sus propias particularidades. El conocimiento y simplificación de la información de éstos fue parte importante del trabajo, mencionando que aún falta mucha información sobre la evolución geológica. También se obtuvo el modelo de elevación digital, interpretando los rasgos morfogenéticos y la distribución de la red de drenaje, así como la delimitación de las subcuencas, en los diferentes campos volcánicos. El trabajo de campo se centró más en la región de Epazoyucan, Singuilucan y Tulancingo, dado los alcances del presente estudio.

Se observa en el área varias subcuencas: Las Navajas, Epazoyucan, Singuilucan, Tecocomulco y Tulancingo (Figura 2). Las corrientes fluviales son intermitentes y presentan varios tipos de patrones de la red de drenaje. Así, la subcuenca Las Navajas presenta un drenaje de tipo radial centrífugo, debido a una gran elevación del complejo estratovolcánico, las subcuencas Epazoyucan y Singuilucan un drenaje de tipo subdendrítico a paralelo, la subcuenca Tecocomulco, un drenaje de tipo subparalelo y la subcuenca Tulancingo, un drenaje de tipo dendrítico y radial (Figura 2). Este tipo de drenaje se corresponde bien con la estructura geológica y la litología, ya que el primero pertenece a un complejo de domos, flujos de lava y piroclastos del Pleistoceno, con predominio de riolitas; el caso de las subcuencas Epazoyucan y Singuilucan se correlacionan a calderas volcánicas del Mioceno (Epazoyucan, Chichicuautla), domos y flujos con predominio de variada litología volcánica-piroclástica de tipo básico, intermedio y ácido. Valadez-Cabrera (2012) [19] reconoce en el complejo volcánico Xihuingo-La Paila, localizado en la subcuenca de Singuilucan, estructuras volcánicas del Mioceno y del Pleistoceno, como son: conos de escoria con flujos de lava asociados, volcanes escudo, domos dacíticos y afloramientos aislados de ignimbritas. 
La subcuenca Tecocomulco con predominio de rocas volcánicas con baja permeabilidad, escasa vegetación y pendientes fuertes. Huizar-Álvarez et al. (2001) [22] establecen que esta subcuenca corresponde a una fosa tectónica de orientación NE-SO que se amplía en el extremo NE, con una litología de sedimentos aluviales y volcanoclásticos, así como rocas volcánicas de composición ácida a básica del Mioceno al Holoceno. Hay fracturamiento de orientación NE-SO, NO-SE y E-O. García-Palomo y Macías (2005) [24], por otra parte, establecieron que las estructuras reconocidas en esta subcuenca son volcanes monogenéticos, domos, mesetas volcánicas y estratovolcanes que representan diferentes ciclos eruptivos. Las fallas orientadas NNE-SSO inclinadas al NO con geometría de tipo semifosa, forman montañas en bloque delimitadas por planicies aluviales. Durante el Pleistoceno se formó la laguna de Tecocomulco, que se considera del tipo vulcano-tectónico [24].

Finalmente, la subcuenca Tulancingo con la presencia de un complejo de domos riolíticos (El Yolo) y dos calderas (Acoculco y Tulancingo) del Plioceno y Pleistoceno.

El control estructural está gobernado por fallas con orientación preferencial NE-SO, que forman bloques tectónicos de tipo fosas (fosa de Apan) y pilares (pilar Cerro Gordo-Las Navajas, pilar Rosario-Acoculco) (Figura 2) [16, 17 y 20].

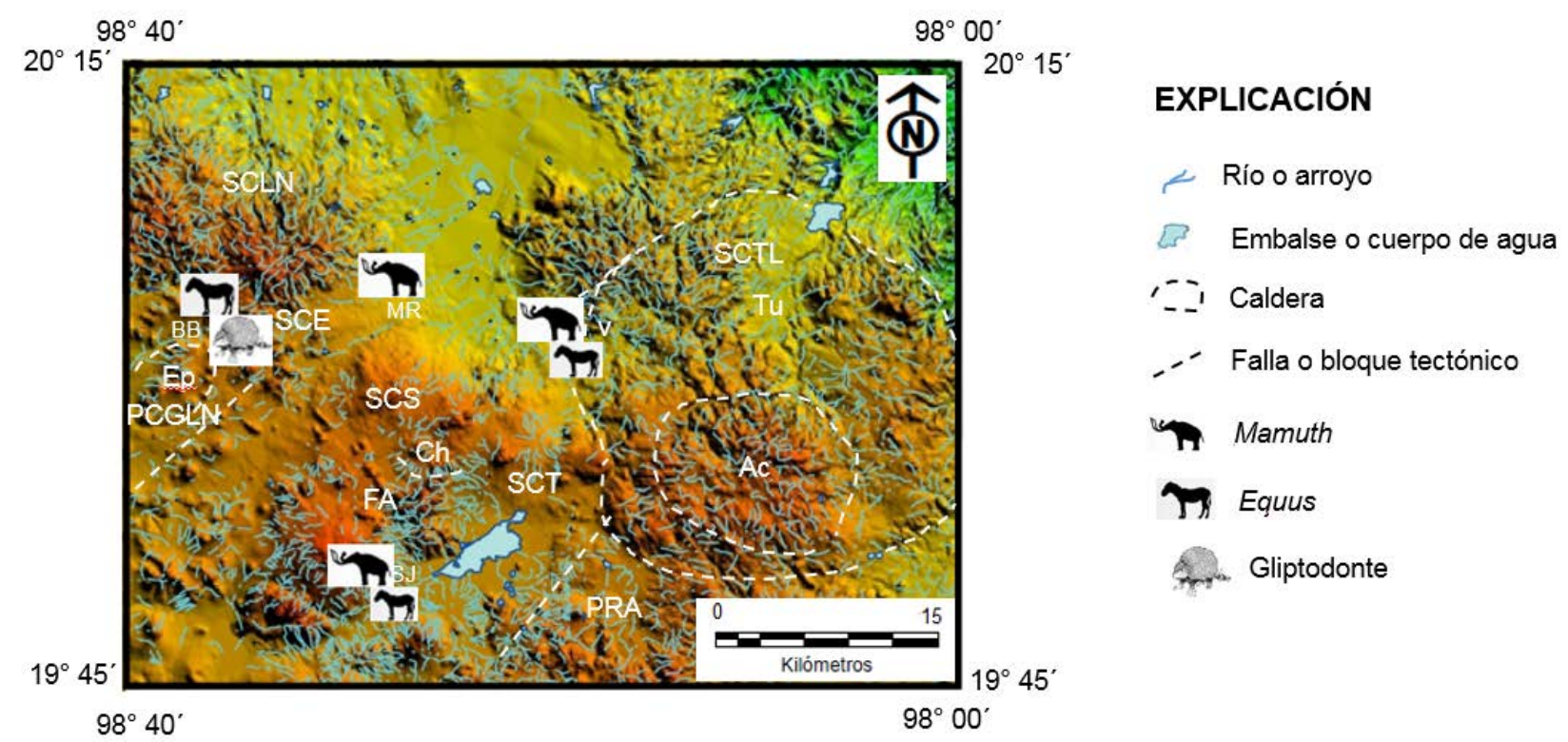

Figura 2. Esquema mostrando el modelo digital de elevación donde se ha sobrepuesto la distribución de la mega-fauna fósil, la red hidrográfica y subcuencas (límites aproximados), así como las calderas y fallas principales.

BB=Barranca El Berrendo; MR=Matías Rodríguez; V=Ventoquipa; SJ=San Jerónimo.

Calderas principales: Ep=Epazoyucan (Mioceno); Ch=Chichicuautla (Mioceno Medio-Tardío); Tu=Tulancingo (Plioceno tardío); Ac=Acoculco (Plioceno tardío-Pleistoceno).

SCLN=Subcuenca las Navajas, SCE= Subcuenca Epazoyucan, SCS= Subcuenca Singuilucan, SCT = Subcuenca Tecocomulco, SCTL= Subcuenca Tulancingo.

Fallas o bloques tectónicos principales: PCGLN=Pilar Cerro Gordo-Las Navajas; FA=Fosa de Apan; PRA=Pilar Rosario-Acoculco.

De la Figura 2 se observa que: 
-Hay una correspondencia entre el borde o centro de algunas calderas y la distribución de embalses o remanentes de cuerpos de agua principales. Esto se debe a la topografía resultante del colapso o al escurrimiento hacia los bordes de estas estructuras volcánicas. Similar situación es apreciable también en el complejo estratovolcánico riolítico de la Sierra de Las Navajas, ya que algunos cuerpos de agua se presentan al norte de la estructura volcánica, donde se emplazó un depósito de avalancha de escombros [12 y13], hace alrededor de $2 \mathrm{Ma}$ [12].

- Los afloramientos de mega-fauna se encuentran en la subcuencas Epazoyucan, Singuilucan, Tecocomulco y Tulancingo.

- El patrón de drenaje es sobre todo de tipo dendrítico a subdendrítico debido a corrientes cortas e irregulares en un terreno volcánico homogéneo y por el control de tipo estructural, por efecto de las fallas que afectan a los complejos volcánicos. La mayor densidad de drenaje es notoria en la subcuenca Tecocomulco.

- La distancia entre los afloramientos de mega-fauna pleistocénica es próxima a los remanentes de cuerpos de agua principales, por ejemplo: Matías Rodríguez dista $2 \mathrm{~km}$ de la presa San Joaquín y $1 \mathrm{~km}$ de dos cuerpos menores en El Sustito; Ventoquipa está a $3 \mathrm{~km}$ de la presa La Esperanza y San Jerónimo a $5.5 \mathrm{~km}$ de la laguna de Tecocomulco. Esto se debe a que la mega-fauna pleistocénica, sobre todo Mammuthus, requerían de grandes cantidades de agua para beber, de algún cuerpo hídrico cercano.

- Existen tramos rectilíneos NE-SO y NO-SE y angulosidad en la configuración del drenaje, lo que es un reflejo de la estructura en bloques de la región.

\section{CONCLUSIONES}

La mega-fauna pleistocénica de la porción meridional del Estado de Hidalgo (región Epazoyucan-Tecocomulco), se distribuye en varios campos volcánicos del Mioceno y del Pleistoceno-Cuaternario. El vulcanismo, además de obturar los cauces naturales, generó variadas estructuras (derrames de lava, domos, calderas, conos cineríticos y estratovolcanes), que aunado al régimen tectónico prevaleciente, modelaron los diferentes tipos de patrones de drenaje, con llanuras extensas por los bloques fallados, siendo propicio al desarrollo de cuerpos hídricos y suelos fértiles que suministraron el vital líquido y alimento para el florecimiento de la mega-fauna pleistocénica que habitó esta porción de México central. En la actualidad, esos cuerpos hídricos quedan únicamente como pequeños embalses o cauces hídricos remanentes.

La mayor densidad de drenaje se localiza en la subcuenca de Tecocomulco. La laguna de esta subcuenca, es posiblemente el vestigio de un gran lago o de un enorme sistema lacustre interconectado de esta porción de la cuenca de México, dado que ha tenido una mayor extensión al curso de su evolución [25].

El régimen extensional con formación de cuencas endorreicas y fosas tectónicas, fue propicio al desarrollo de lagos y ríos, depositándose los sedimentos fluviales y lacustres de la Formación Tarango o Calpulalpan, dónde se hallan los restos de la mega-fauna fósil del Pleistoceno tardío. 


\section{BIBLIOGRAFÍA}

[1] GeomapApp, Marine Geoscience Data System. Columbia University. http://www.geomapapp.org/. 2014.

[2] V.M. Bravo-Cuevas, E. Ortiz-Caballero, M.A. Cabral-Perdomo. Bol. Soc. Geol. Mex. 61, 2 (2009) 267-76.

[3] V.M. Bravo-Cuevas, E. Jiménez-Hidalgo, J. Priego-Vargas. Rev. Mex. Cien. Geol. 28, 1 (2011) 65-82.

[4] M.A. Cabral-Perdomo. Universidad Autónoma del Estado de Hidalgo, Instituto de Ciencias Sociales y Humanidades, Pachuca, Hidalgo 1 (2010) 13-21.

[5] C. Camacho, La Jornada, México D.F. Domingo 26 de octubre de 2003. https://www.jornada.com.mx/2003/10/26/05an2cul.php?origen=cultura.php\&fly=

[6] V.M. Bravo-Cuevas, N., M. Morales-García, M.A. Cabral-Perdomo. Bol. Soc. Geol. Mex. 67, 2 (2015) 337-47.

[7] E. Monroy-Rodríguez, D. Contreras-Cruz, A. Palma-Ramírez, R.G. Martínez-Serrano. Geos 351 (2015).

[8] B.B. Ramírez-Ramírez. Instituto Politécnico Nacional, ESIA-Unidad Ticomán, tesis de Licenciatura, México, D.F. (2016) 157 p.

[9] D. Contreras-Cruz, O. Aparicio-Canales, R., G. Martínez-Serrano, E. MonroyRodríguez, G.P. García-Tovar. Geos 351 (2015).

[10] O. Aparicio-Canales, D. Contreras-Cruz. Universidad Autónoma del Estado de Hidalgo, ICBI, tesis de Licenciatura, Pachuca, Hidalgo (2016) 116 p.

[11] G.P. García-Tovar, R.G. Martínez-Serrano, J. Solé, J.C. Correa-Tello, E.Y. NúñezCastillo, H. Guillou, E. Monroy-Rodríguez. Rev. Mex. Cien. Geol. 32 (2015) 100-22.

[12] S.A. Nelson, A. Lighthart, 1997, II Convención sobre la Evolución Geológica de México y Recursos Asociados, Pachuca, Hidalgo (1997) 89-96.

[13] M.V. Núñez-Velázquez, R.G. Martínez-Serrano, D. Contreras-Cruz, G. SolísPichardo, P. Girón-García. Geos 371 (2017).

[14] A. Castro-García, D. Córdoba-Méndez. Universidad Autónoma del Estado de Hidalgo, Pancromo 23, 1 (1994) 26-39.

[15] R.E Sánchez, P.M. Osorio. Geociencia 3 (2008) 5-24.

[16] A. García-Palomo, J. L. Macías, G. Tolson, G. Valdez, J.C. Mora, 2002, Geof. Int. 41, 2 (2002) 133-50.

[17] A. García-Palomo, J. L. Macías, A. Jiménez, G. Tolson, M. Mena, J.M. SánchezNúñez, J.L. Arce, P.W. Layer, M.A. Santoyo, J. Lermo-Samaniego. Jour. Volcan. Geoth. Res. 349 (2018) 240-55.

[18] K. Juárez-López. Universidad Nacional Autónoma de México, tesis de Maestría, México, D.F. (2015) 128 p.

[19] S. Valadez-Cabrera. Universidad Nacional Autónoma de México, tesis de Maestría, México, D.F. (2012) 161-5

[20] A. López-Hernández. Universidad Nacional Autónoma de México, tesis de Doctorado, México, D.F. (2009) 170 p.

[21] D.R. Avellán, J.L. Macías, P.W. Layer, G. Cisneros, J.M. Sánchez-Núñez, M.G. Gómez-Vasconcelos, A. Pola, G.S. Ceballos, F. García-Tenorio, G. Reyes-Agustín, S., Osorio-Ocampo, L. García-Sánchez, I.F. Mendiola, J. Martí, H. López-Loera, J. Benowitz. Jour. Maps (2018) 1-11. 
[22] R. Huizar-Álvarez, O. Campos-Enríquez, L.M. Mitre-Salazar, D. Alatriste-Vilchis, T. Méndez-García, J. Juárez-Sánchez. Rev. Mex. Cien. Geol. 181 (2001) 55-73.

[23] O. Ledezma-Guerrero. Universidad Nacional Autónoma de México, Instituto de Geología, Carta Geológica de México, serie 1:100, 000 (1987), mapa con texto, 12 p.

[24] A. García-Palomo, J.L. Macías. Universidad Nacional Autónoma de México, Instituto de Geología. R. Huizar-Álvarez, E.J. Jiménez-Fernández, C. Juárez-López, eds., Publicación especial 3, México, D.F. (2005) 9-20.

[25] M. Caballero, S. Lozano, B. Ortega, J. Urrutia, J.L. Macías. Universidad Nacional Autónoma de México, Instituto de Geología. R. Huizar-Álvarez, E.J. JiménezFernández, C. Juárez-López, eds., Publicación especial 3, México, D.F. (2005) 4971. 